\title{
Dimensional and metric structures in multidimensional stimuli
}

\author{
WILLA KAY WIENER-EHRLICH \\ Brown University, Providence, Rhode Island 02912
}

\begin{abstract}
The present experiments investigated two characteristics of subjects' multidimensional representations: their dimensional organization and metric structure, for both analyzable and integral stimuli. In Experiment 1, subjects judged the dissimilarity between all pairs of stimuli differing in brightness and size (analyzable stimuli), while in Experiment 2, subjects made dissimilarity judgments for stimuli varying in width height, and area shape (integral stimuli). For the brightness size stimuli, the findings that (a) brightness judgments were independent of size (and vice versa) and (b) the best fitting scaling solution was one that depicted an orthogonal structure are strong evidence that subjects perceived brightness size as a dimensionally organized structure. In contrast, for the rectangle stimuli, neither width height nor area shape contributed additively to overall dissimilarity. The results of the metric fitting were more equivocal. For all stimulus sets, the Euclidean metric yielded scaling solutions with lower stress values than the city block metric. When bidimensional ratings were regressed on unidimensional ratings, the city block metric yielded a slightly higher correlation coefficient than the Euclidean metric for brightness size stimuli. The two rules of combination were equivalent for the width-height stimuli, but the Euclidean metric provided a better approximation for the area shape stimuli. The results were discussed in terms of how subjects integrate physical dimensions for the case of integral stimuli and the superiority of dimensional vs. metric structure as an indicator of stimulus analyzability.
\end{abstract}

Much research with multidimensional stimuli is based on the assumption that such stimuli are perceived and organized in a dimensional fashion. However, recent studies suggest that the decomposition of stimuli into the component dimensions that generate them may be possible for only some multidimensional stimuli. For example, color patches varying in terms of brightness, hue, and saturation are processed in a wholistic scan and therefore the global properties of the stimulus are emphasized over the underlying constituent dimensions. Since such stimuli cannot be easily analyzed into their components, they produce interference when a subject must selectively attend to one dimension of an orthogonally varying stimulus set and ignore (or filter) the irrelevant dimensions (Egeth \& Pachella, 1969; Felfoldy, 1974; Garner \& Felfoldy, 1970; Gottwald \& Garner, 1975; Pomerantz \& Garner, 1973; Pomerantz \& Schwaitzberg, 1975) but yield an improvement in processing speed when both dimensions are correlated and perceptual integration

This article is based on research conducted by the author when she was a Postdoctoral Research Fellow at Brown University. The work was supported principally by a postdoctoral fellowship from the National Institute of Health (Grant MH-00235). I gratefully acknowledge the assistance of Barbara Burns and Bryan Shepp and would like to thank them for their many helpful suggestions and comments. Requests for reprints should be sent to Willa Kay Wiener-Ehrlich, Butler Hospital, Department of Research and Evaluation, 345 Blackstone Boulevard, Providence, Rhode Island 02906. is desirable (Eriksen \& Hake, 1955; Garner \& Felfoldy, 1970; Lockhead \& King, 1977; Monahan \& Lockhead, 1977) or when both dimensions must be attended to anyway (Lockhead \& King, 1977; Monahan \& Lockhead, 1977; Pomerantz \& Schwaitzberg, 1975).

Furthermore, because unanalyzable stimuli are perceived as differing on only one complex dimension, subjects can make judgements of perceived similarity directly. Hence they yield Euclidean metrics in direct distance scaling (Indow \& Kanazawa, 1960; Indow \& Uchizono, 1960; Kruskal, 1964) and similarity structures in free classification experiments (Handel, 1967; Handel \& Imai, 1972) where both dimensional and similarity classifications are possible. Thus, the hallmark of nonanalyzable stimuli is the dominance of similarity relations over dimensional organization.

In contrast, schematic faces varying in terms of shape of face, empty or filled eyes, and type of mouth (Tversky \& Krantz, 1969), parallelograms varying in size and tilt (Attneave, 1950; Hyman \& Well, 1967), brightness and size stimuli (Attneave, 1950; Gottwald \& Garner, 1975; Handel \& Imai, 1972), and stimuli varying in color and form (Gottwald \& Garner, 1972) are analyzed into constituent dimensions, and each dimension can selectively engage the attention of the visual scanner. Since subjects can selectively attend to component dimensions, variation in the irrelevant dimension 
does not interfere with the processing of the relevant dimension, and there is no loss in processing speed with orthogonally varying stimuli (Garner \& Felfoldy, 1970; Gottwald \& Garner, 1972, 1975; Imai \& Garner, 1965). At the same time, however, because perceptual fusion or integration is less likely with highly analyzable stimuli, subjects cannot make use of dimensional redundancy to improve their classification performance (Garner \& Felfoldy, 1970), and they find the processing of information from both dimensions (e.g., assigning red circles and blue squares to one category and blue circles and red squares to another) to be quite difficult (Gottwald \& Garner, 1972, 1975).

The fact that selective attention is possible with such stimuli has two additional consequences. First, dimensional classifications predominate in free classification experiments, and dimensional preferences can occur (Imai, 1966; Imai \& Garner, 1965). Second, when subjects are asked to give similarity ratings, they do so by summing distances along each dimension. Thus, a city block metric provides a close approximation to subjects' proximity ratings (Shepard, 1964). Stimuli perceived as homogeneous, unitary wholes are termed integral stimuli, while those stimuli that are analyzed into perceptually distinct parts are called analyzable stimuli. Analyzable stimuli are perceived as dimensionally organized structures, while integral stimuli are not.

A more formal approach to dimensional structure, based on properties fundamental to subjective dimensions, is embodied in the additive difference model of dimensional organization (Beals, Krantz, \& Tversky, 1968; Krantz \& Tversky, 1975; Tversky \& Krantz, 1969, 1970). The first necessary condition for a psychological dimension is a kind of contextindependent property, that it be independent of the level of the other dimension. If width and height are psychologically valid dimensions of figures, then similarity judgments of figures that differ in width should be independent of the height of the figure. This property is referred to as interdimensional additivity and requires that the distance between items be a function of the sum of their differences on the constituent dimensions. A second basic property of subjective dimensions is that of intradimensional subtractivity, in which the contribution of any single dimension to similarity depends on the absolute difference between the two stimuli on that dimension. It should be emphasized that this approach treats dimensional models as quantitative psychological theories that can be confirmed by certain patterns in the similarity data.

The present experiments incorporate Krantz and Tversky's measurement theoretic techniques to compare subjects' representations of integral vs. analyzable stimuli. First, several product structures thought to be representative of analyzable and integral stimuli were constructed. In Experiment 1, subjects judged the dissimilarity between all pairs of stimuli differing in brightness and size (analyzable stimuli), while in Experiment 2, subjects made dissimilarity ratings for stimuli varying in width and height, and area and shape (integral stimuli). These dissimilarity ratings were then used to diagnose subjective dimensions as follows. The judgments were first examined for interdimensional additivity. (Predictions derivable from the axiom are discussed below.) It was expected that the analyzable stimuli would exhibit this property while integral stimuli would not. The best fitting metric was then examined for the two stimulus types. Since independence of subjective dimensions (interdimensional additivity) is one characteristic of stimulus analyzability, stimuli that exhibit interdimensional additivity should be best represented by a city block metric, while stimuli that do not (i.e., integral stimuli) should be best represented by the Euclidean metric.

\section{Stimulus Structure}

In the present experiments, the coordinate systems are restricted to only two dimensions and to four levels on each dimension. Thus, as shown in Figure 1, one of the stimulus sets is represented as a $4 \times 4$ structure in perceived brightness and size. For each structure, 120 dissimilarity ratings corresponding to a subject's perceived distance of 120 stimulus pairs were collected. Since predictions from the interdimensional additivity axiom are stated in terms of stimulus pairs, the following notation, taken from Krantz and Tversky (1975), is introduced to

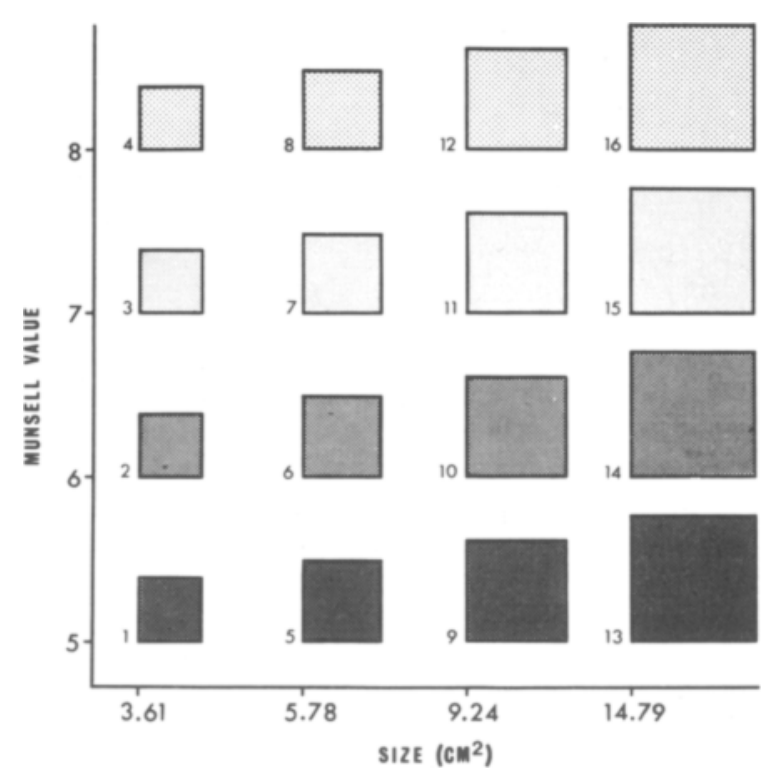

Figure 1. The 16 figures from Experiment 1 plotted in log area and Munsell value coordinates. 
describe the stimulus pairs. (The reader will find the notation easier to follow if he refers to Figure 1.) Brightness and size are the canonical dimensions; the notation is similar for width height and area shape.

Pairs of brightness (size) levels will be referred to as $B(S)$ intervals. Atomic intervals will be denoted as $B_{1}, B_{2}, B_{3},\left(S_{1}, S_{2}, S_{3}\right)$; hence, $B_{1}=\left(b_{1}, b_{2}\right)=$ $\left(b_{5}, b_{6}\right)$ and $B_{3}=\left(b_{3}, b_{4}\right)=\left(b_{7}, b_{8}\right)$ [and $S_{1}=\left(s_{1}\right.$, $\left.s_{s}\right)=\left(s_{2}, s_{6}\right)$ and $\left.S_{3}=\left(s_{9}, s_{13}\right)=\left(s_{10}, s_{14}\right)\right]$. Adjacent intervals can combine, and these combined intervals are denoted by subscripts. For example, $B_{12}\left(S_{12}\right)$ denotes intervals $B_{1}$ and $B_{2}\left(S_{1}\right.$ and $\left.S_{2}\right) \quad\left[B_{12}=\right.$ $\left.\left.\left(b_{1}, b_{3}\right)=\left(b_{5}, b_{7}\right), S_{12}=\left(s_{1}, s_{9}\right)=s_{2}, s_{10}\right)\right] ; B_{23}\left(S_{23}\right)$ denotes intervals $B_{2}$ and $B_{3}\left(S_{2}\right.$ and $\left.S_{3}\right)\left[B_{23}=\left(b_{2}\right.\right.$, $\left.\left.\left.b_{4}\right)=\left(b_{6}, b_{8}\right), S_{23}=\left(s_{5}, s_{13}\right)=s_{6}, s_{14}\right)\right]$; and $B_{123}$ $\left(S_{123}\right)$ denotes intervals $B_{1}, B_{2}, B_{3}\left(S_{1}, S_{2}, S_{3}\right)\left[B_{123}=\right.$ $\left.\left(b_{1}, b_{4}\right)=\left(b_{5}, b_{8}\right), S_{123}=\left(s_{1}, s_{13}\right)=\left(s_{2}, s_{14}\right)\right]$. The total number of different intervals for a dimension is seven $B_{0}, B_{1}, B_{2}, B_{3}, B_{12}, B_{23}, B_{123}$ and $S_{0}, S_{1}, S_{2}, S_{3}$, $\mathrm{S}_{12}, \mathrm{~S}_{23}, \mathrm{~S}_{123}$.

If the 120 stimulus pairs are considered as elements in a factorial design whose factors are brightness intervals and size intervals, that design can be represented as in Table 1. Note that this design is an unbalanced one. As Table 1 indicates, there are four stimulus pairs for cells that exhibit undimen- sional variation but only two stimulus pairs in the bidimensional cells.

\section{Interdimensional Additivity}

Interdimensional additivity asserts that the contribution of different dimensions to overall dissimilarity is additive, so that dissimilarity can be decomposed into dissimilarity of component parts. (The particular type of additive combination rule is left unspecified.) Two sets of predictions are derivable from the interdimensional additivity axiom. The first set of predictions, called the equality predictions, is easily explained in terms of the interval notation introduced earlier: Any stimulus pair designated by the same interval notation should yield equal dissimilarity ratings. For example, the four stimulus pairs $(1,13),(2,14),(3,15),(14,16)$ differ by the same interval notation along the size dimension, but at increasing brightness value. Since they differ by the same interval along each dimension (i.e., a $S_{123}$ interval on the size dimension and no difference, $B_{0}$, on the brightness dimension), they all have the same interval notation. Hence, their dissimilarity rating should be equal. Pairs occupying the same cell in Table 1 should thus have equal dissimilarity ratings.

The second set of predictions is called the order-

Table 1

The 120 Stimulus Pairs Arranged in Brightness Interval by Size Interval Matrix, Experiment 1

\begin{tabular}{|c|c|c|c|c|c|c|c|}
\hline \multirow{2}{*}{$\begin{array}{c}\text { Size } \\
\text { Interval }\end{array}$} & \multicolumn{7}{|c|}{ Brightness Interval } \\
\hline & $\mathbf{B}_{0}$ & $\mathbf{B}_{1}$ & $\mathrm{~B}_{2}$ & $\mathrm{~B}_{3}$ & $B_{12}$ & $B_{23}$ & $B_{123}$ \\
\hline$S_{0}$ & & $\begin{array}{r}1,2 \\
5,6 \\
9,10 \\
13,14\end{array}$ & $\begin{array}{r}2,3 \\
6,7 \\
10,11 \\
14,15\end{array}$ & $\begin{array}{r}3,4 \\
7,8 \\
11,12 \\
15,16\end{array}$ & $\begin{array}{r}1,3 \\
5,7 \\
9,11 \\
13,15\end{array}$ & $\begin{array}{r}2,4 \\
6,8 \\
10,12 \\
14,16\end{array}$ & $\begin{array}{r}1,4 \\
5,8 \\
9,12 \\
13,16\end{array}$ \\
\hline$S_{1}$ & $\begin{array}{l}1,5 \\
2,6 \\
3,7 \\
4,8\end{array}$ & $\begin{array}{l}1,6 \\
2,5\end{array}$ & $\begin{array}{l}2,7 \\
3,6\end{array}$ & $\begin{array}{l}3,8 \\
4,7\end{array}$ & $\begin{array}{l}1,7 \\
3,5\end{array}$ & $\begin{array}{l}2,8 \\
4,6\end{array}$ & $\begin{array}{l}1,8 \\
4,5\end{array}$ \\
\hline$S_{2}$ & $\begin{array}{l}5,9 \\
6,10 \\
7,11 \\
8,12\end{array}$ & $\begin{array}{l}5,10 \\
6,9\end{array}$ & $\begin{array}{l}6,11 \\
7,10\end{array}$ & $\begin{array}{l}7,12 \\
8,11\end{array}$ & $\begin{array}{l}5,11 \\
7,9\end{array}$ & $\begin{array}{l}6,12 \\
8,10\end{array}$ & $\begin{array}{l}5,12 \\
8,9\end{array}$ \\
\hline $\mathrm{S}_{3}$ & $\begin{array}{r}9,13 \\
10,14 \\
11,15 \\
12,16\end{array}$ & $\begin{array}{r}9,14 \\
10,13\end{array}$ & $\begin{array}{l}10,15 \\
10,14\end{array}$ & $\begin{array}{l}11,16 \\
12,15\end{array}$ & $\begin{array}{r}9,15 \\
11,13\end{array}$ & $\begin{array}{l}10,16 \\
12,14\end{array}$ & $\begin{array}{r}9,16 \\
12,13\end{array}$ \\
\hline$S_{12}$ & $\begin{array}{l}1,9 \\
2,10 \\
3,11 \\
4,12\end{array}$ & $\begin{array}{l}1,10 \\
2,9\end{array}$ & $\begin{array}{l}2,11 \\
3,10\end{array}$ & $\begin{array}{l}3,12 \\
4,11\end{array}$ & $\begin{array}{l}1,11 \\
3,9\end{array}$ & $\begin{array}{l}2,12 \\
4,10\end{array}$ & $\begin{array}{l}1,12 \\
4,9\end{array}$ \\
\hline$S_{23}$ & $\begin{array}{l}5,13 \\
6,14 \\
7,15 \\
8,16\end{array}$ & $\begin{array}{l}5,14 \\
6,13\end{array}$ & $\begin{array}{l}6,15 \\
7,14\end{array}$ & $\begin{array}{l}7,16 \\
8,15\end{array}$ & $\begin{array}{l}5,15 \\
7,13\end{array}$ & $\begin{array}{l}6,16 \\
8,14\end{array}$ & $\begin{array}{l}5,16 \\
8,13\end{array}$ \\
\hline$S_{123}$ & $\begin{array}{l}1,13 \\
2,14 \\
3,15 \\
4,16\end{array}$ & $\begin{array}{l}1,14 \\
2,13\end{array}$ & $\begin{array}{l}2,15 \\
3,14\end{array}$ & $\begin{array}{l}3,16 \\
4,15\end{array}$ & $\begin{array}{l}1,15 \\
3,13\end{array}$ & $\begin{array}{l}2,16 \\
4,14\end{array}$ & $\begin{array}{l}1,16 \\
4,13\end{array}$ \\
\hline
\end{tabular}


ing predictions. Empirically, this would mean that a given disimilarity ordering of intervals along one dimension is preserved when adding any interval from a second dimension to each of them, and vice versa. For example, if the psychological dissimilarity, $\delta$, of interval $S_{2}$ of the size dimension is greater than the psychological dissimilarity of interval $S_{1}$ [i.e., $\delta\left(S_{2}\right)>\delta\left(S_{1}\right)$ ], then the effect of adding a second interval, $B_{3}$, to $S_{1}$ and $S_{2}$ should be to maintain the ordinal relationship between $S_{1}$ and $S_{2}\left[\right.$ i.e., $\left.\delta\left(B_{3} S_{2}\right)>\delta\left(B_{3} S_{1}\right)\right]$.

\section{EXPERIMENT 1}

\begin{abstract}
Method
Subjects

The subjects were 10 undergraduate students enrolled at Brown University. There were four male and six female subjects. All subjects completed two 50 -min sessions and were paid $\$ 4$ for their participation.
\end{abstract}

\section{Stimuli}

The stimuli were 16 achromatic squares, cut from glossy Munsell paper, that varied both in size (area) and Munsell value (brightness). The Munsell system divides luminous reflectance into 11 perceptually equal steps called Munsell values, with a perfect black as 0 and a perfect white as 10 . Thus, equal increments on the Munsell scale correspond to equal increments in perceived brightness. The scale yielding equal spacing among Munsell values is calibrated for Munsell chips presented under a standard light source (i.e., artificial daylight that has a correlated color temperature of $6,700^{\circ} \mathrm{K}$ ) against an intermediate gray background (Newhall, 1940). Therefore, the present experiment employed artificial daylight fluorescent bulbs and, as background for the Munsell chips, an intermediate gray matte surface, approximating a Munsell value of 5 . The surface on which the stimulus pairs were placed was also covered with matte paper, approximating the Munsell level of 5.

To make the size dimension comparable with the brightness dimension, adjacent levels of the size dimension must correspond to equal increments in perceived size. A logarithmic manipulation of the size (area) continuum was introduced to achieve the goal of perceptually equal size intervals. The a priori effectiveness of this manipulation is suggested by the following findings: (a) Attneave (1950) had indicated that the psychological distance between squares varying in area from 6.45 to $40.32 \mathrm{~cm}^{2}$ is a linear function of difference in logarithm of area. (b) Krantz and Tversky (1975) provided scaling data for rectangles varying in area and shape that indicated that logarithmic spacing was effective in inducing the perception of equal area intervals.

In Figure 1, each square is represented as a point in a size brightness coordinate system. The size dimenson is given in log area coordinates, where the ratio between the adjacent levels is 1.6 .

\section{Procedure}

Each subject was presented with a sequence of 120 achromatic Munsell chips and asked to indicate the degree of dissimilarity between the two figures on a 10-point scale. The order of presentation was randomized for each subject and for each session. Before each session, subjects were read the following instructions: "This is an experiment in mental organization. The procedure is quite simple. You are being asked to perform a type of psychological task called a dissimilarity task. In this experiment, we will show you pairs of figures and we'll ask you to indicate, on a ten-point scale, the degree of dissimilarity between the two figures. For example, if the figures are almost identical, that is, the dissimilarity between them is very small, use that part of the scale marked 1 . If the figures are very different from one another, use that part of the scale marked 10. In the same fashion, for all intermediate levels of dissimilarity between the figures, use the intermediate values of the scale depending on the judged degree of dissimilarity. We are interested in your subjective impression of degree of dissimilarity. Different people are likely to have different impressions, so there are no correct or incorrect answers. Simply look at the pair of figures for a short time, and use the number that corresponds to the degree of dissimilarity between the figures."

Throughout the session, subjects were reminded that low numbers should correspond to low dissimilarity and high numbers to high dissimilarity. Reference to dimensional structure of the dimensions brightness and size were avoided.

The subjects were run individually in a session that lasted for $50 \mathrm{~min}$. The subjects were required to attend two sessions, where the two sessions occurred within 2 days of each other.

\section{Design}

Table 1 presents the 120 brightness size stimulus pairs in a factorial design whose factors are brightness intervals and size intervals. Since each subject was presented with all 120 stimulus pairs over both sessions, the design was a complete within-subject factorial design with the factors Brightness (B), Size (S), Time $(T)$, Subjects $(K)$, and Stimulus Pairs (P). Stimulus pairs were nested with the factors Brightness and Size $[P(B \times S)]$ and crossed with Subjects and Time $[P(B \times S) \times K \times T]$.

\section{Results}

A basic assumption is that within the psychological space, the stimuli can be decomposed into dimensions where the distances between adjacent levels are equal. The first goal is to discover the psychophysical function that describes the mapping of the physical input onto each of the dimensions; second, to determine whether the 16 stimuli are embedded in a dimensionally organized space; and third, to see which metric best describes the psychological space.

\section{Psychophysical Function}

If the reader examines the 24 stimulus pairs that vary along a single continuum, he can observe that there are only six different types of variation. Several of these intervals are the same step size (e.g., $B_{1}$, $\mathbf{B}_{2}$, and $\mathbf{B}_{3}$ ), and they can be grouped together. Thus, if the unidimensional scales contain perceptually equal steps, then (a) intervals that are of the same step size should have the same dissimilarity ratings and (b) the dissimilarity ratings should increase linearly with interval step size.

The left-hand panel of Figure 2 presents the mean dissimilarity ratings (averaged over time and subject) of stimulus pairs as a function of difference in logarithm of area, with brightness held constant. The right-hand panel presents the average dissimilarity rating of stimulus pairs as a function of difference in Munsell value, with area held constant. Note that the two functions are approximately linear; the mean dissimilarity ratings do increase at a constant rate as a function of interval size. However, intervals that 

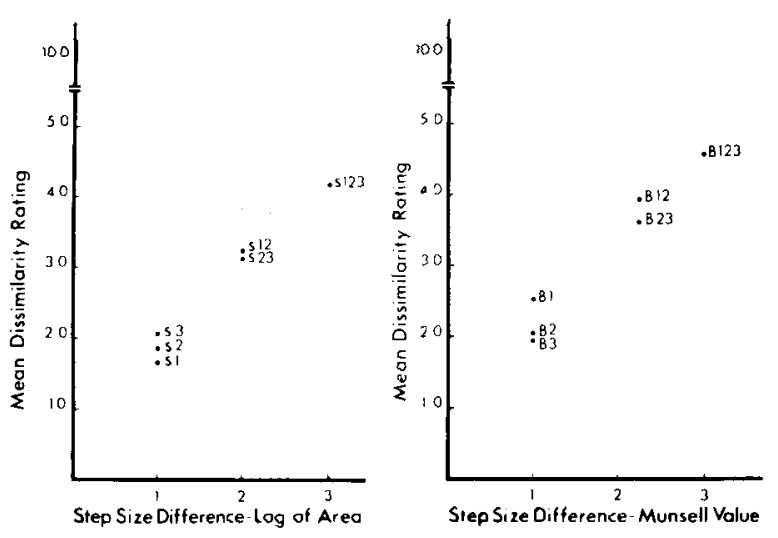

Figure 2. Psychological difference between Munsell squares as a function of difference in logaritbm of area with Munsell value constant (left panel) and as a function of difference in Munsell value with area constant (right panel).

are of the same step size do not have the same mean dissimilarity rating. This visual observation of Figure 2 is borne out by an analysis of the difference in mean ratings of theoretically equivalent intervals. Interval $\mathbf{B}_{\mathbf{1}}$ is more dissimilar than interval $\mathbf{B}_{\mathbf{2}}$ $[F(1,45)=6.07, p<.025]$, and interval $S_{3}$ is more dissimilar than the mean of intervals $S_{1}$ and $S_{2}$ $[F(1,45)=4.25, p<.05]$. One can conclude, then, that the differences between dimension levels are not equivalent. Thus, for these stimuli, the psychophysical relationship is not given by a simple logarithmic transformation (for size), or by a power function relating perceived brightness and luminous reflectance (the relation between Munsell values and luminous reflectance, Krantz, 1972).

\section{Dimensional Representation}

Analysis of variance. As indicated previously, two sets of predictions are derivable from the interdimensonal additivity axiom. Both sets of predictions have straightforward rules of correspondence to a standard factorial design. In terms of an analysis of variance, the equality prediction is equivalent to a nonsignificant stimulus pairs effect [i.e., $P(B \times S)$ ] while the ordering prediction is equivalent to a null interaction effect. Since the number of stimulus pairs varies for different brightness size combinations, the Stimulus Pairs effect was analyzed separately for each of 24 stimulus pairs exhibiting unidimensional variation and the 72 stimulus pairs exhibiting bidimensional variation. The degree of invariance of the relationship between intervals within one dimension across intervals of the second dimension (the interaction effect) was analyzed for the 72 stimulus pairs that exhibited bidimensional variation. Stimuli varying in terms of both brightness and size are assumed to be analyzable stimuli, and thus capable of being decomposed into constituent dimensions. The predictions are: (a) unidimensional and bidimensional stimulus pairs should yield a nonsignificant Stimulus Pairs effect and (b) bidimensional stimulus pairs should yield a nonsignificant interaction effect when analyzed in terms of their mean dissimilarity ratings.

The Stimulus Pairs effect was nonsignificant for all three analyses. Apparently, then, any fixed interval on one dimension contributed approximately the same amount to dissimilarity irrespective of the level of the orthogonal dimension, confirming the equality prediction. The interaction effect was nonsignificant for stimulus pairs varying in terms of both brightness and size. The null interaction effect is illustrated in Figure 3. Each set of six curves shows the effect of varying differences in one dimension, with difference on the other dimension held constant
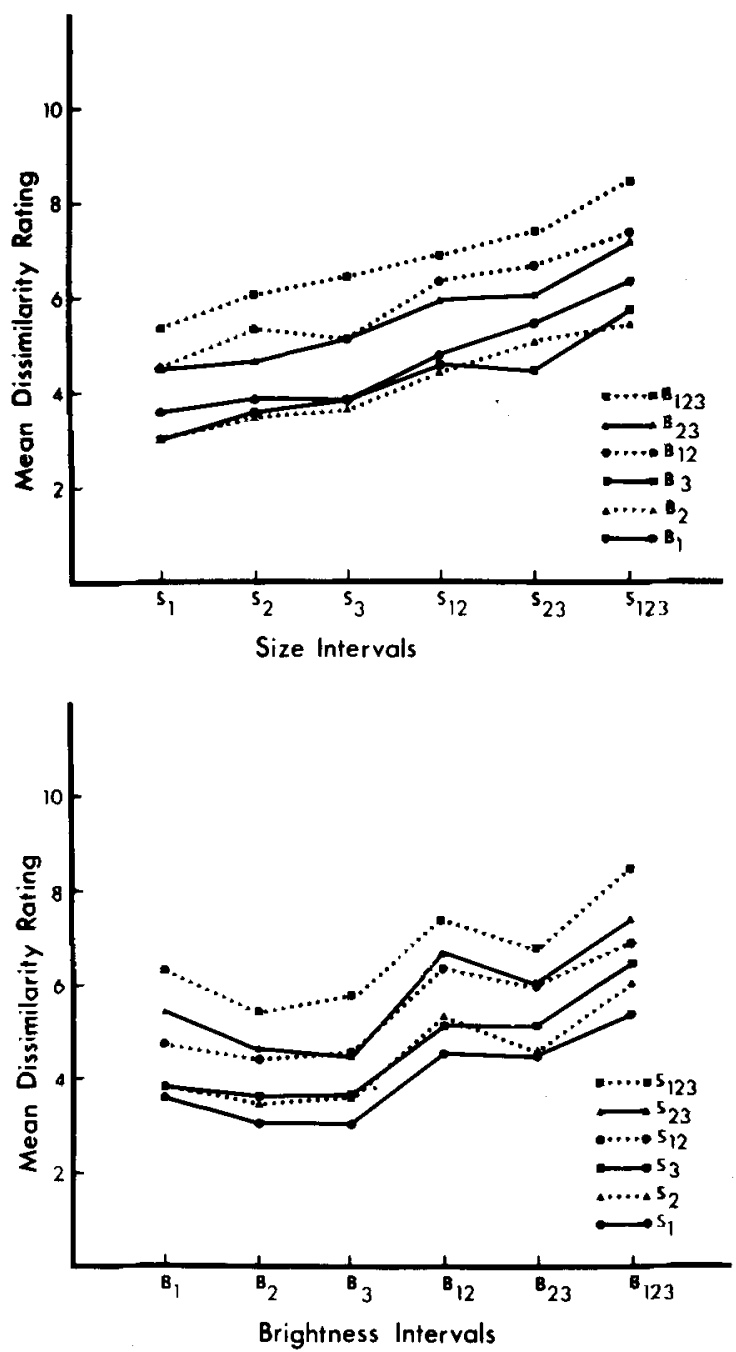

Figure 3. Psychological difference between Munsell squares as a function of difference in logarithm of area, with difference in Munsell value held constant at six values (top panel) and as a function of difference in Munsell value, with difference in area beld constant at six values (bottom panel). 
at some value. The top panel of Figure 3 presents the mean dissimilarity ratings between squares, averaged over time and subject, as a function of difference in the logarithm of area, with brightness held constant at six interval values. The bottom panel presents a similar comparison with the dimensions reversed. Casual inspection of these curves show that these functions are parallel. It can be concluded that brightness and size contribute additively to determine overall dissimilarity.

Note, however, that the invariance of dissimilarity scores for one dimension across levels of the second dimension refers to the invariance of the ordinal relationships between stimulus scores. A nonparametric analysis of variance, the Friedman rank order test (Bradley, 1976; Winer, 1971) was used to test for the presence of an interaction. A chi square equal to $29.06(\mathrm{df}=25)$ was obtained, which was not significant at the .05 level of significance. The data therefore do not contradict the hypothesis of an invariant ranking of intervals on one dimension across different intervals of the second dimension.

Multidimensional scaling. A representation of Munsell squares in a two-dimensional space was obtained by applying Kruskal's (1964) MDSCAL IV program (Version 5MS) to the mean dissimilarity ratings of the 120 stimulus pairs. One widely known difficulty with this method is the problem of arriving at a local minimum rather than the true overall minimum. To reduce the possibility of obtaining a solution that is a local minimum solution, the data were analyzed from 10 different random starting configurations (Arabie, 1973). The best fitting configuration is presented in Figure 4. Since the same solution (up to changes in rotation and reflection) occurred several times and had a stress value that was considerably less than the value found for the

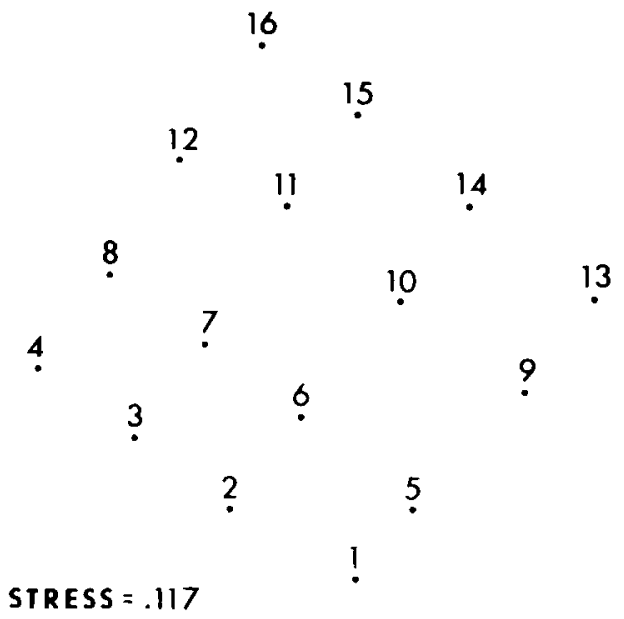

Figure 4. Best fitting scaling configuration for the 16 Munsell squares (Experiment 1). (This configuration is based on a random starting configuration, Euclidean metric, secondary approach to ties and Stress Formula 1.) other solutions, it appears that it is the true minimum solution.

Inspection of Figure 4 shows that brightness sizes are orthogonal dimensions. It should be pointed out, however, that according to Kruskal's system of labels for particular values of stress (Kruskal, 1964), the goodness of fit of the final configuration to subjects' proximity data (stress $=.117$ ) is only fair. The stress value is but one of the many pieces of evidence that can be used in interpreting scaling results. Since the stimuli's spatial arrangement is theoretically interpretable, one can conclude that the obtained solution is valid and not heavily influenced by factors such as random fluctuations in the data, degeneracy, and/or a merely local minimum.

Figure 4 also provides information relevant to the function relating perceived brightness to Munsell value. If the reader compares intervals $B_{1}$ and $B_{2}$ of the brightness dimension, he can observe that $B_{1}$ is psychologically more dissimilar than $B_{2}$ (i.e., stimulus pairs differing by a $B_{1}$ interval are spatially more distant than stimulus pairs differing by $\mathbf{B}_{2}$ ). Note that the greater dissimilarity of $\mathbf{B}_{1}$ over $B_{2}$ is maintained across all levels of the size dimension. Thus, the Munsell spacing on the brightness dimension did not result in subjectively equal intervals.

\section{Metric Fitting}

The data up to this point converge on a common finding that subjects employed the additive combination rule in their judgments of bidimensional variation. This section provides a more precise characterization of the additive combination rule by analyzing how the dissimilarities along each dimension combine to determine overall dissimilarity. Since brightness size dimensions appear to be easily isolated perceptually, subjects should judge the stimulus distance separately for each dimension, and then combine the distances to get an overall distance. The city block metric should therefore yield a solution with the lowest stress.

Multidimensional scaling. For each of the two Minkowski metrics under consideration, Euclidean vs. city block, the mean dissimilarity ratings were analyzed in 10 different scaling runs. Each scaling solution was based on a random starting configuration and secondary approach to ties. In all cases, the Euclidean configuration of metric distances provided the best fit to the data in terms of Stress Formula 1 (stress $=.117$ to .272 , median value $=$ .117 for Euclidean; stress $=.158$ to .363 , median value $=.334$ for city block). Furthermore, since the "best" configuration (theoretically most interpretable configuration) was selected from among the Euclidean scaling runs, it seems that the underlying rule of combination is best approximated by an Euclidean metric. The city block is, of course, the 
metric one would expect if subjects were judging the dissimilarity on each dimension independently, and then combining the dissimilarities to get an overall subjective dissimilarity.

The above analysis assumes that different Minkowski solutions are equally robust under deviations from the posited metric. Shepard (1974), however, cites results that indicate that "purely Euclidean solutions can be surprisingly robust in the face of certain kinds of rather marked departures from the assumed Euclidean metric" (p. 407). Thus, the present pattern of results may be an artifact of the nonmetric multidimensional scaling procedure, namely, that minor discrepancies in the true underlying metric are of greater consequence to the city block vs. the Euclidean solution. This consideration suggests a change in the method of metric fitting.

Multiple regression analysis. To determine which of the two rules of combination is most appropriate, for each rule of combination, the mean dissimilarity ratings corresponding to the 120 stimulus pairs were regressed on the dissimilarity judgments of onedimensional differences. The goodness of fit of the two rules of combination was assessed by comparing the resulting multiple correlation coefficients.

For the city block metric,

$$
\dot{D_{i j}}=\sum_{k=1}^{K}\left(w_{k}^{*}\left|x_{i j}-x_{j k}\right|\right),
$$

the prediction equation takes the form

$$
Y_{p}=w_{b} B_{i}+w_{s} S_{i^{\prime}}+c .
$$

For the Euclidean metric,

$$
D_{i j}=\left[\sum_{k=1}^{K} w_{k}^{*}\left(x_{i k}-x_{j k}\right)^{2}\right]^{1 / 2},
$$

the prediction equation takes the form

$$
Y_{p}{ }^{2}=w_{b} B_{i}{ }^{2}+w_{s} S_{i^{2}}{ }^{2}+c .
$$

Fitting these equations by the method of least squares, we obtain

$$
Y_{p}=.87 B_{i}+.87 S_{i^{\prime}}+.23
$$

and

$$
\mathrm{Y}_{\mathrm{p}}{ }^{2}=1.59 \mathrm{~B}_{\mathrm{i}}{ }^{2}+1.84 \mathrm{~S}_{\mathrm{i}^{\prime}}{ }^{2}-4.37 .
$$

These equations indicate that the variables of brightness and size are equally effective in determining psychological distance. The multiple correlation coefficient is .983 for the city block metric (implying that $97 \%$ of the variance in the $\mathrm{Y}$ values was explained) and the multiple correlation coefficient is .961 for the Euclidean metric (implying that $92 \%$ of the variance in the $\mathrm{Y}^{2}$ values was explained). These results indicate that the city block metric provides only a marginally better approximation to subjects' rule of combination than the Euclidean metric.

\section{Discussion}

The present experiment started off by asstiming that the 16 stimuli could be described by a vector of values on each of two dimensions, and that an ordinal measure of dissimilarity between stimuli was given. The next step was to uncover configuration of metric distances, primarily by analyzing the ordering of these dissimilarities. The finding that perceived differences along the two dimensions contributed additively to overall dissimilarity implies independent dimensions and is consistent with the posited dimensional structure. The finding that the dissimilarities conformed to a city block metric agrees with the dimensions' perceptual distinctiveness. Taken together, these two findings support a multidimensional metric model of the stimulus space.

Support for a dimensional analysis was based on the fact that judgements of the brightness of figures were independent of the size of the figures, and vice versa. The nonsignificant Stimulus Pairs effect indicated that a pair of brightness (or size) levels defined the same interval regardless of the level of the orthogonal dimension. The finding of a nonsignificant interaction effect indicated that the ordering of brightness (or size) intervals was preserved by adding a common size (or brightness) interval to them. Furthermore, since the best fitting solution to the dissimilarity data was one that depicted an orthogonal structure, there is strong evidence that the 16 stimuli were perceived as a dimensionally organized structure.

Support for the city block metric was more equivocal. The city block metric yielded higher stress values and less interpretable configurations as compared to solutions obtained for the Euclidean metric. since the Euclidean metric is more robust under violations of metric assumptions than other Minkowski $r$ metrics, suboptimal or local minimum solutions may be more likely to occur with city block and other nonEuclidean solutions. When the brightness size data were rescaled using a different iterative procedure, the MINISSA-I/SSA-I algorithm (Lingoes, 1973), a Kruskal stress value of .119 was obtained for the Euclidean metric and a Kruskal stress value of .043 for the city block metric. Apparently, the Guttman Lingoes SSA-I algorithm is a better procedure for avoiding suboptimal 
solutions (Spence, 1972; Lingoes \& Roskam, Note 1), either because its rational initial configuration reduces the likelihood of being trapped in a nonoptimal position or because its iterative procedure (double-phase rank image procedure preceding a single-phase procedure) ignores local minima when far from the minimum solution. Furthermore, since in the multiple regression analysis, the city block metric yielded a slightly higher multiple correlation coefficient than the Euclidean rule of combination, it appears that the metric approximates a city block one.

The present experiment is the first study that the author knows of to contrast the distance metric underlying a set of multidimensional stimuli with an explicit measure of the stimuli's dimensional structure. Although Tversky and Krantz (1969) investigated interdimensional additivity for schematic faces, they did not test the goodness of fit of a variety of metric models. (They indicate, however, that a city block metric would not give a good fit to their mean ratings, notwithstanding their stimuli's analyzability.) Handel and Imai (1972) used a derived measure of dimensional organization, classification structure in a free classification task, to contrast with the best fitting distance metric. Although these investigators were able to pinpoint the metric underlying brightness size stimuli as a city block one (in contrast to our demonstration of only an approximation toward the city block metric), the present experiment provides a more precise measure of dimensional structure than their classification analysis.

A strong empirical association between dimensional organization and the underlying metric was reported by Hyman and Well (1967). They indicate that dimensional interaction was greater for the homogeneous color patches than for the more analyzable geometric stimuli (Attneave parallelograms and Shepard circles). Furthermore, within each stimulus set, the degree of interaction was correlated with the metric's deviation from the city block and toward the dominance end of the Minkowski continuum. Note that, in contrast to this experiment, Hyman and Well tested only one dimensional additivity prediction, that of interstimulus distance equality.

Taken as a whole, then, the results suggest a dependency between the dimensional organization underlying a stimulus set and the particular combination rule subjects use for bidimensional variation. It still remains to be determined whether stimuli that are reacted to as homogeneous, unanalyzable wholes exhibit both an Euclidean metric and a nondimensional structure when analyzed according to the additive difference axioms. The remainder of the paper is addressed to the relationship between dimensional structures and Minkowski metrics for unitary (integral) stimuli.

\section{EXPERIMENT 2}

The present experiment considers two alternative product structures of rectangles, width and height, and area and shape to determine which, if either, satisfies the interdimensional additivity axiom of the additive difference model. Although brightness size stimuli can be characterized naturally in terms of multiple subjective attributes, dimensional structures appear to be less relevant to form perception. Wender (1971) collected dissimilarity ratings of rectangles varying in area and shape, in order to determine whether they satisfied decomposability and intradimensional subtractivity. His results indicated an interaction between the area and shape dimensions: an increase in dissimilarity produced by a given area interval as the shape became more extreme. Felfoldy (1974) used a speeded classification paradigm to investigate how subjects integrated width and height. He reported that there was significant interference produced with orthogonally varying stimuli when the task was to sort by a given dimension. Krantz and Tversky (1975) performed a formal analysis of both product structures using ordinal dissimilarity judgments. They indicate that for both sets, there was a tendency for an interval along one dimension to appear larger, the higher or more extreme the level of the orthogonal dimension. (The effect, however, was more pronounced for the area shape structure.)

These findings indicate that rectangles should be especially good exemplars of homogeneous, wholistic stimuli. Hence, they should provide a sensitive test of the relationship between dimensional structure and metric representations for the case of integral stimuli.

Subjects

\section{Method}

The subjects were 10 undergraduate students enrolled at Brown University. There were five male and five female subjects. AII subjects completed two $11 / 2-h$ sessions and were paid $\$ 8$ for their participation.

\section{Stimuli}

The stimuli were 32 white rectangles on an intermediate gray background. In Figure 5, each rectangle is plotted in log width $x$ log height coordinates, in which the ration between adjacent levels along the width height dimensions is 1.3. Note that the 32 rectangles are simultaneously members of a second dimensional structure, area shape. (This structure is represented in Figure 5 by dashed lines at $45^{\circ}$ to the width height structure.) Thus, the stimuli form the larger part of an incomplete $7 \times 7$ factorial structure in width height and a complete $4 \times 4$ structure in perceived area shape.

Krantz and Tversky (1975) indicate that a logarithmic spacing yielded approximately equal spacing for both sets of dimensions, and therefore a logarithmic manipulation was employed in the 


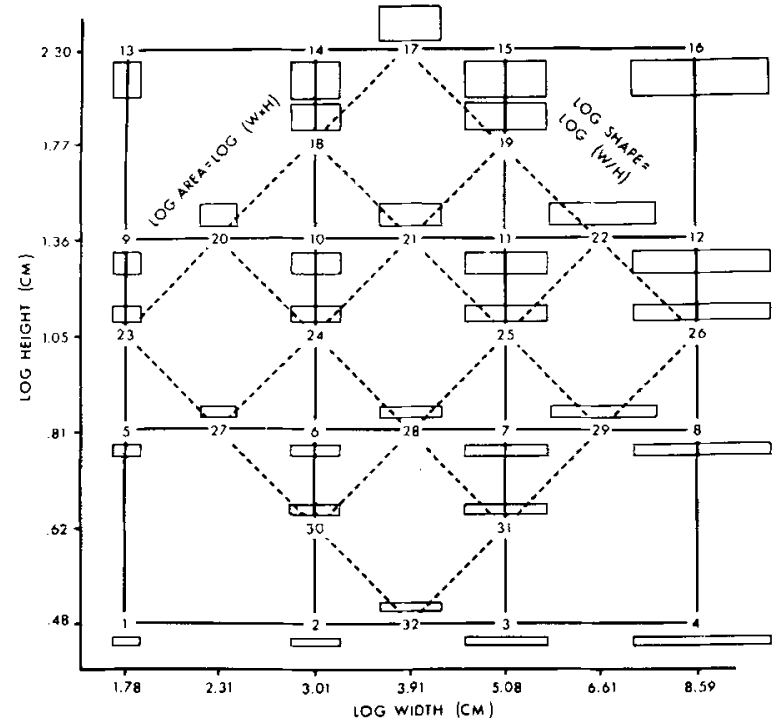

Figure 5. The 32 figures from Experiment 2 plotted in $\log$ width and $\log$ height coordinates. (The $\log$ area by $\log$ shape coordiuates are represented by dashed lines at $45^{\circ}$ to the width height structure.)

current experiment. Krantz and Tversky also report that a significant area illusion existed in the perception of rectangle size: Tall rectangles looked bigger than shorter rectangles of equal area. To avoid this, they selected rectangles to be squat, so that the largest height value was smaller than the smallest width value.
In the present experiment, the largest height value was approximately equal to the next-to-the-smallest width value.

To reduce the complexity of subsequent analyses, as well as to insure that the present dimensional structures were equivalent to the $4 \times 4$ brightness size structure, the 32 rectangles were divided into two sets of 16 stimuli. Stimuli 1-16 corresponded to a $4 \times 4$ width-height coordinate system, while Stimuli 17-32 corresponded to a $4 \times 4$ area-shape coordinate system. There are 120 stimulus pairs for each product structure and a total of 240 stimulus pairs. Dissimilarity judgments were analyzed separately for each product structure.

Notation. The same notation used for brightness size stimuli will be used for pairs of area levels, shape levels, widt levels, and height levels. There are seven different intervals on each dimension. The three atomic $\mathbf{A}$ intervals will be denoted as $\mathbf{A}_{1}$, $\mathbf{A}_{2}, \mathbf{A}_{3}$, proceeding from low area to high area. Thus, $\mathbf{A}_{\mathbf{1}}=$ $\left(a_{23}, a_{20}\right)$ and $A_{3}=\left(a_{18}, a_{11}\right)$. Combination of adjacent intervals are represented as $A_{12}=\left(a_{23}, a_{18}\right), A_{23}=\left(a_{20}, a_{17}\right)$, and $A_{123}=$ $\left(a_{23}, a_{17}\right)$. In terms of $S$ intervals, $S_{1}=\left(s_{17}, s_{19}\right), S_{12}=\left(s_{17}, s_{22}\right)$, etc. Similar notation is introduced for $\mathbf{H}$ and $W$ intervals: $H_{1}=\left(h_{1}, h_{5}\right), \quad H_{23}=\left(h_{5}, h_{13}\right) ; \quad$ and $\quad W_{1}=\left(W_{1}, W_{2}\right), \quad W_{12}=$ $\left(w_{1}, w_{3}\right)$.

\section{Procedure}

The subjects were presented with a random sequence of 240 stimulus pairs (120 stimulus pairs from each product structure) and asked to indicate the degree of dissimilarity on a 10-point scale. Low numbers corresponded to low dissimilarity and high numbers corresponded to high dissimilarity. Before each session, the subjects were read the same instructions used in Experiment 1. Reference to dimensional structure was scrupulously avoided.

The subjects were run individually in a session that lasted for about $90 \mathrm{~min}$. The subjects attended two sessions that occurred approximately within 2 days of each other. The order of pre-

Table 2

The 120 Stimulus Pairs Arranged in Area Interval by Shape Interval Matrix, Experiment 2

\begin{tabular}{|c|c|c|c|c|c|c|c|}
\hline \multirow{2}{*}{$\begin{array}{c}\text { Area } \\
\text { Interval }\end{array}$} & \multicolumn{7}{|c|}{ Shape Intervals } \\
\hline & $S_{0}$ & $S_{1}$ & $S_{2}$ & $S_{3}$ & $S_{12}$ & $S_{23}$ & $S_{123}$ \\
\hline$A_{0}$ & & $\begin{array}{l}17,19 \\
18,21 \\
20,24 \\
23,27\end{array}$ & $\begin{array}{l}19,22 \\
21,25 \\
24,28 \\
27,30\end{array}$ & $\begin{array}{l}22,26 \\
25,29 \\
28,31 \\
30,32\end{array}$ & $\begin{array}{l}17,22 \\
18,25 \\
20,28 \\
23,30\end{array}$ & $\begin{array}{l}19,26 \\
21,29 \\
24,31 \\
27,32\end{array}$ & $\begin{array}{l}17,26 \\
18,29 \\
20,31 \\
23,32\end{array}$ \\
\hline$A_{1}$ & $\begin{array}{l}23,20 \\
27,24 \\
30,28 \\
32,31\end{array}$ & $\begin{array}{l}23,24 \\
27,20\end{array}$ & $\begin{array}{l}27,28 \\
30,24\end{array}$ & $\begin{array}{l}30,31 \\
32,28\end{array}$ & $\begin{array}{l}23,28 \\
30,20\end{array}$ & $\begin{array}{l}27,31 \\
32,24\end{array}$ & $\begin{array}{l}23,31 \\
32,20\end{array}$ \\
\hline$A_{2}$ & $\begin{array}{l}20,18 \\
24,21 \\
28,25 \\
31,29\end{array}$ & $\begin{array}{l}20,21 \\
18,24\end{array}$ & $\begin{array}{l}24,15 \\
28,21\end{array}$ & $\begin{array}{l}28,29 \\
31,25\end{array}$ & $\begin{array}{l}20,25 \\
28,18\end{array}$ & $\begin{array}{l}24,29 \\
31,21\end{array}$ & $\begin{array}{l}20,29 \\
31,18\end{array}$ \\
\hline $\mathbf{A}_{3}$ & $\begin{array}{l}18,17 \\
21,28 \\
25,22 \\
29,26\end{array}$ & $\begin{array}{l}18,19 \\
17,21\end{array}$ & $\begin{array}{l}21,22 \\
19,25\end{array}$ & $\begin{array}{l}15,26 \\
29,22\end{array}$ & $\begin{array}{l}18,22 \\
25,17\end{array}$ & $\begin{array}{l}21,26 \\
29,19\end{array}$ & $\begin{array}{l}18,26 \\
29 ; 17\end{array}$ \\
\hline$A_{22}$ & $\begin{array}{l}23,18 \\
27,21 \\
30,25 \\
32,29\end{array}$ & $\begin{array}{l}23,21 \\
27,18\end{array}$ & $\begin{array}{l}27,25 \\
30,21\end{array}$ & $\begin{array}{l}30,29 \\
32,25\end{array}$ & $\begin{array}{l}23,25 \\
30,18\end{array}$ & $\begin{array}{l}27,29 \\
32,21\end{array}$ & $\begin{array}{l}23,29 \\
32,18\end{array}$ \\
\hline$A_{23}$ & $\begin{array}{l}20,17 \\
24,19 \\
28,22 \\
31,26\end{array}$ & $\begin{array}{l}20,19 \\
17,24\end{array}$ & $\begin{array}{l}24,22 \\
19,28\end{array}$ & $\begin{array}{l}28,26 \\
22,31\end{array}$ & $\begin{array}{l}20,22 \\
17,28\end{array}$ & $\begin{array}{l}24,26 \\
31,19\end{array}$ & $\begin{array}{l}20,26 \\
31,17\end{array}$ \\
\hline$A_{123}$ & $\begin{array}{l}23,17 \\
27,19 \\
30,22 \\
32,26\end{array}$ & $\begin{array}{l}23,19 \\
17,27\end{array}$ & $\begin{array}{l}27,22 \\
30,19\end{array}$ & $\begin{array}{l}30,26 \\
22,32\end{array}$ & $\begin{array}{l}23,22 \\
17,30\end{array}$ & $\begin{array}{l}27,26 \\
19,32\end{array}$ & $\begin{array}{l}23,26 \\
32,17\end{array}$ \\
\hline
\end{tabular}


sentation of rectangle pairs was randomized for each subject and for each session.

\section{Design}

If the 120 pairs of rectangles corresponding to a given dimensional structure are considered as elements in a complete $7 \times 7$ factorial design, whose factors are width intervals and height intervals (or area intervals and shape intervals), that design can be represented as in Tables 1 and 2 . Substituting width (W) for brightness (B) and height $(\mathrm{H})$ for size (S), Table 1 presents the 120 width-height stimulus pairs in a Width Interval by Height Interval matrix. Table 2 presents the 120 area shape stimulus pairs in an Area Interval by Shape Interval matrix. Since each subject was presented with all rectangle pairs, for both sessions, the design was a within-subject factors design, with the factors Width (W), Height (H) [or Area (A), Shape (S)], Time (T), Subjects $(K)$, and Stimulus Pairs (P). Stimulus pairs were nested within the two dimension factors $[P(W \times H), P(A \times S)]$, and crossed with Subjects and Time $[\mathbf{P}(\mathbf{W} \times \mathbf{H}) \times \mathbf{K} \times \mathbf{T}$ and $\mathbf{P}(\mathbf{A} \times \mathbf{S})$ $\times \mathrm{K} \times \mathrm{T}]$. As in Experiment 1, the design was an unbalanced one. Thus, there are four levels of the Stimulus Pair factor for stimuli exhibiting unidimensional variation, and only two levels of the Stimulus Pair factor for stimuli exhibiting bidimensional variation.

\section{Psychophysical Function}

\section{Results}

Krantz and Tversky (1975) report that a logarithmic manipulation yielded perceptually equal spacing for both sets of factors. Thus, the present experiment should also be successful in developing unidimensional scales with perceptually equal steps. The predictions are (a) equal-sized intervals (i.e., $\mathbf{W}_{12}, \mathbf{W}_{23}$ ) should receive the same mean dissimilarity rating and (b) mean dissimilarity ratings should increase linearly with interval size.

Figures 6 and 7 present mean dissimilarity as a function of difference in logarithm of one dimension, with the orthogonal dimension held constant. Each figure is based on the ratings obtained from the 24 rectangle pairs that appear either in the first row or first column of Tables 1 and 2 . The ratings were averaged over time and subject, and then the mean
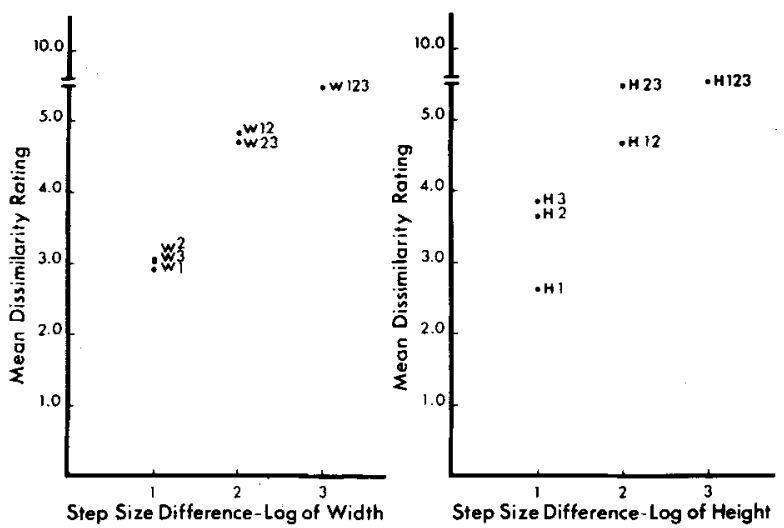

Figure 6. Psychological difference between rectangles as a function of difference in logarithm of width with height constant (left panel) and as a function of difference in logarithm of height with width constant (right panel).
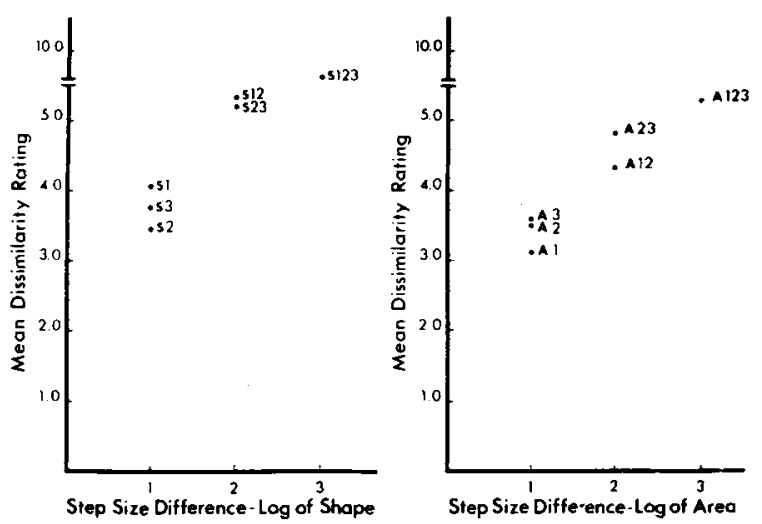

Figure 7. Psychological difference between rectangles as a function of difference in logarithm of shape $n$ ith area constant (left panel) and as a function of difference in logarithm of area with shape constant (right panel).

ratings of the four stimulus pairs within a given interval were obtained. The left-hand panel of Figure 6 presents dissimilarity ratings as a function of difference in logarithm of width with height constant. The righthand panel presents a similar analysis with the dimensions reversed. Figure 7 presents dissimilarity as a function of difference in logarithm of shape with area held constant in the left panel; on the right, the same analysis is presented but with the dimensions reversed. Casual inspection of these figures indicates that the functions appear to be reasonably linear for three out of four dimensions. (the only exception is the height dimension). Equal-sized intervals also tended to receive equal dissimilarity ratngs, again with the exception of the height dimension. These observations are borne out by planned comparisons on the ratings of theoretically equivalent intervals. There were no significant differences in the dissimilarity of theoretically equivalent intervals for the width and shape dimensions, and only one significant difference, between $A_{12}$ and $A_{23}[F(1,45)=4.82, p<.05]$ for the area dimension. In contrast, for the height dimension, all three comparisons on equal-sized intervals were significant. Interval $\mathrm{H}_{2}$ was statistically more dissimilar than interval $H_{1}[F(1,45)=8.56$, $\mathrm{p}<.001$ ], interval $\mathrm{H}_{3}$ was statistically more dissimilar than the mean of intervals $H_{1}$ and $H_{2}[F(1,45)=$ $6.21, \mathrm{p}<.025$ ], and interval $\mathrm{H}_{23}$ was more dissimilar than $H_{12}[F(1,45)=15.03, p<.001]$. Thus, the logarithmic manipulation appears to be successful in developing unidimensional scales with perceptually equal intervals for three of four physical dimensions.

\section{Dimensional Representation}

Analysis of variance. Since rectangles may be better characterized as wholistic forms rather than dimensionally derived stimuli, they should not be easily decomposed into their underlying perceptual attributes. Thus, an additive combination rule should 
not apply. Both a significant Stimulus Pairs effect and a significant interaction effect are predicted for width height and area shape product structures.

In order that there be a constant number of stimulus pairs across all interval combinations, the Stimulus Pairs effect was analyzed separately for the unidimensional and bidimensional stimulus pairs. Stimulus pairs varying on both dimensions are needed to test for an interaction, and there fore only the 72 bidimensional pairs were tested for an interaction effect. Thus, there were six separate analysesthree for each product structure.

First, there was a significant Stimulus Pairs effect for both width height and area shape $[F(18,162)=$ $5.91, \mathrm{p}<.001$, for width; $F(18,162)=6.00, \mathrm{p}<.001$, for height; $F(36,324)=5.93, p<.001$, for width height; $F(18,162)=5.47, p<.001$, for area; $F(18,162)$ $=11.31, \mathrm{p}<.001$, for shape; $\mathrm{F}(36,324)=9.33$, $\mathrm{p}<.001$, for area shape] and therefore a violation of the equality prediction. The Stimulus Pairs effect was readily interpretable for one of the dimensional structures, width height. For these rectangles, there was a tendency for the width interval to appear larger, the more extreme the level of the height dimension. For example, $(1,2)$ was less dissimilar than $(5,6)$, which was less dissimilar than $(9,10)$, although all are $\mathrm{W}_{1} \mathrm{H}_{0}$ pairs. Since, as one goes from $(1,2)$ to $(9,10)$, the level of the orthogonal height dimension increases, this effect can be described as an augmentation effect. Although Krantz and Tversky report a pronounced augmentation effect for area and shape, our area shape rectangles failed to exhibit systematic deviations from the equality prediction. Thus, there was no tendency for a pair of shapes to be more dissimilar as their (equal) area value decreased.

Second, the interaction effect was significant for both product structures $[\mathrm{F}(25,225)=5.14, \mathrm{p}<.001$, for width height; $F(25,225)=7.00, p<.001$, for area shape]. The significant interaction effects are illustrated in Figures 8 and 9. Each set of curves shows the effect of varying difference on one dimension (e.g., moving from $W_{1}$ to $W_{123}$ ), with difference in the other dimension held constant (e.g., at $\mathrm{H}_{12}$ ). Figure 8, top panel, presents the dissimilarity ratings between rectangles, averaged over time and subjects, as a function of difference in logarithm of width, with difference in logarithm of height held constant at six interval values. The bottom panel presents a similar comparison, but with the dimensions reversed. The top panel of Figure 9 presents the dissimilarity ratings as a function of difference in logarithm of area with difference in logarithm of shape held constant, and the bottom panel presents the same comparison but with the dimensions reversed. Since the functions with a given family of curves are not parallel, the ordering between intervals on one dimension is dependent on the interval of the second dimension. This con- clusion is supported by the results of a Friedman nonparametric analysis of variance which also yielded significant interaction effects for both structures $\left[\chi^{2}(25)=78.70, \mathrm{p}<.001\right.$, for width height; $\chi^{2}(25)$ $=101.78, \mathrm{p}<.001$, for area shape]. Apparently, then, width height and area shape combine in a nonadditive fashion to determine overall similarity.

Multidimensional scaling. For each product structure, Kruskal's 1964 MDSCAL IV program was applied to the dissimilarity data collected on all possible stimulus pairs. The best fitting twodimensional configurations are presented in Figures 10 and 11. Since the same solution (up to changes in rotation and reflection) occurred several times, the configurations appear to be the "true" underlying configurations.
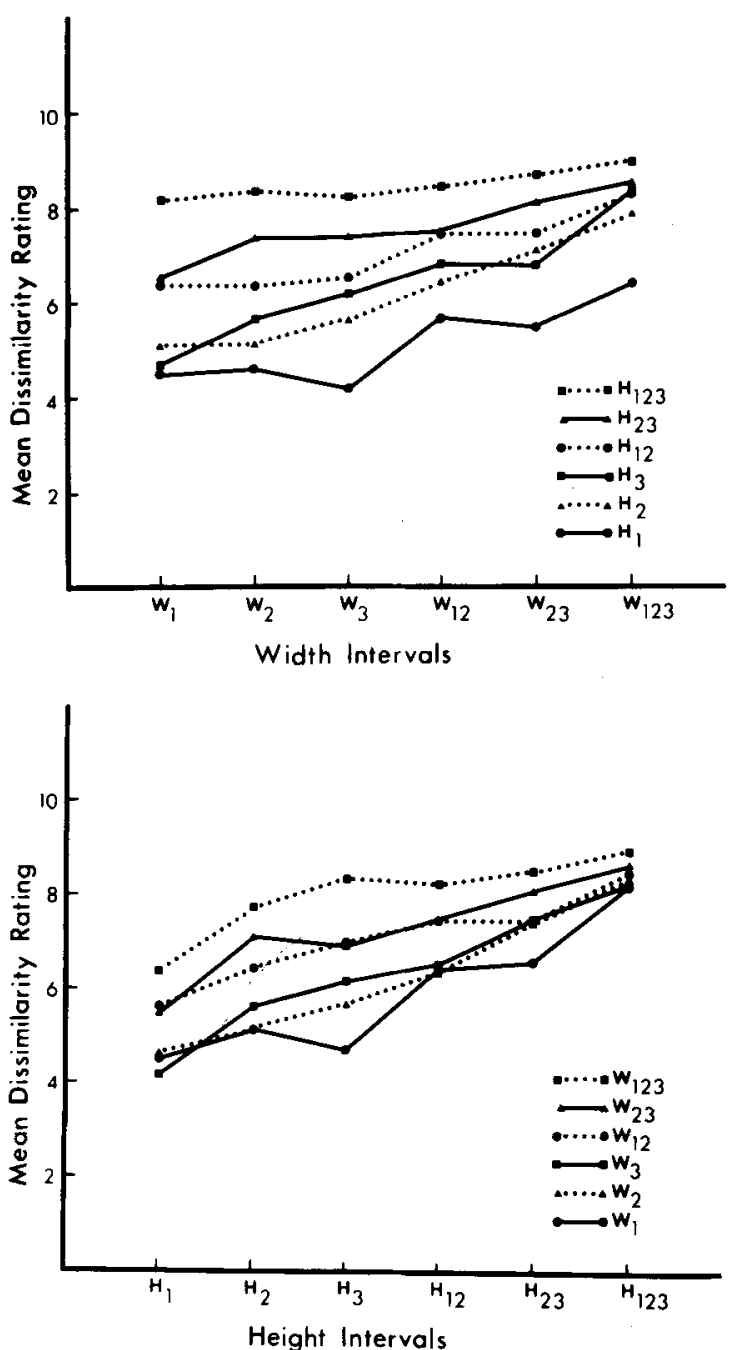

Figure 8. Psychological difference between rectangles as a function of difference in logarithm of width, with difference in Jogarithm of height held constant at six values (top panel), and as a function of difference in logarithm of height, with difference in logarithm of width beld constant at six values (bottom panel). 

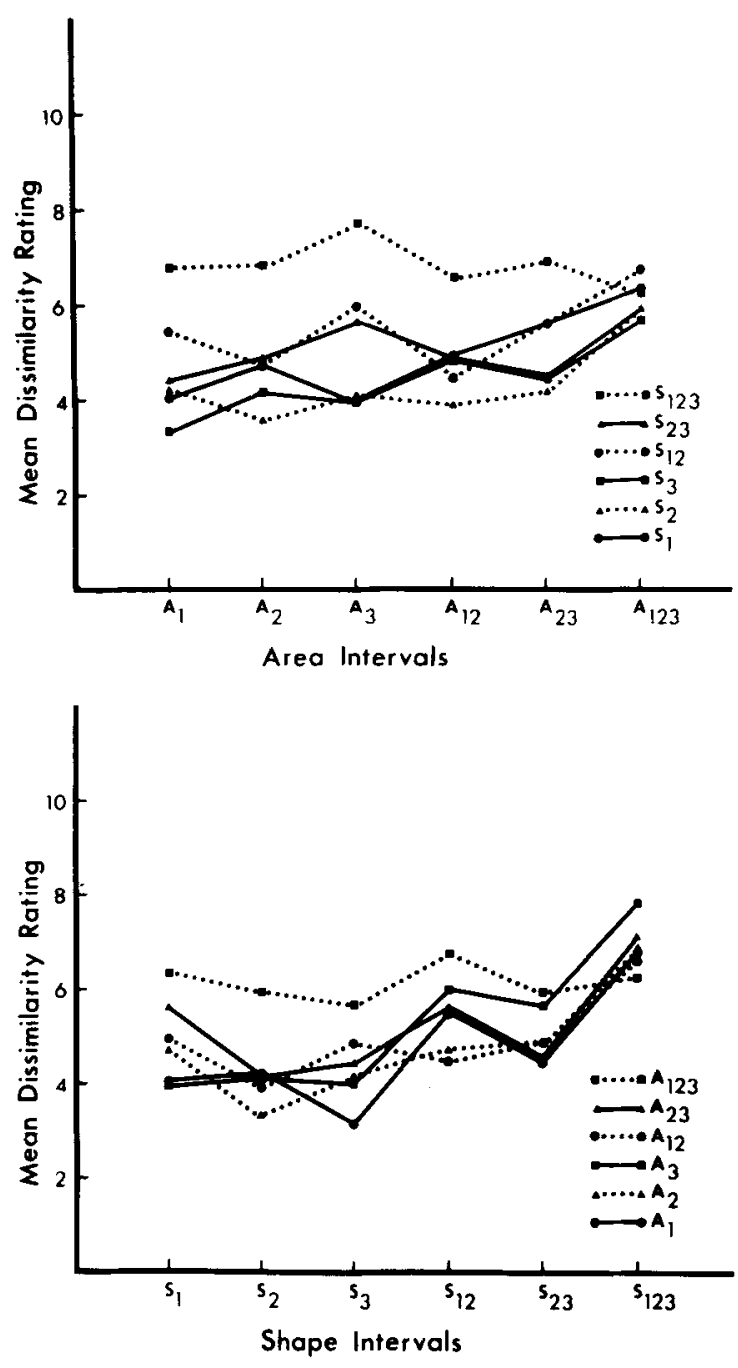

Figure 9. Psychological difference between rectangles as a function of difference in logarithm of area, with difference in logarithm of shape held constant at six values (top panel), and as a function of difference in logarithm of shape, with difference in logarithm of area held constant at six values (bottom panel).

The goodness of fit is only "fair" for both product structures (.105 for width height, .113 for area shape; the expected stress under the hypothesis of a random ordering is .279; see Klahr, 1969). However, the two configurations faithfully capture the interaction between attributes. Furthermore, the augmentation effect is clearly evident in the widthheight structure. Inspection of Figure 10 indicates that same width interval appears larger, the higher the height level. The analogous increase in dissimilarity produced by a given height interval, as width becomes more extreme, is less compelling. There is no evidence of an augmentation effect for the area shape structure. These results are therefore consistent with the results reported in the analyses of variance: namely, interacting dimensions and an augmentation effect for only the width height product structure.

\section{Metric Fitting}

The data up to this point indicate that rectangles are perceived as homogeneous wholistic forms that are not easily decomposed into their underlying constituent components. Since the lack of dimensional structure implies that subjects are unable to attend selectively to dimensions, subjects should view these stimuli as differing on only one complex dimension. Hence, the rectangles should yield Euclidean as opposed to a city block metric in direct distance scaling.

Multidimensional scaling analysis. For each set of

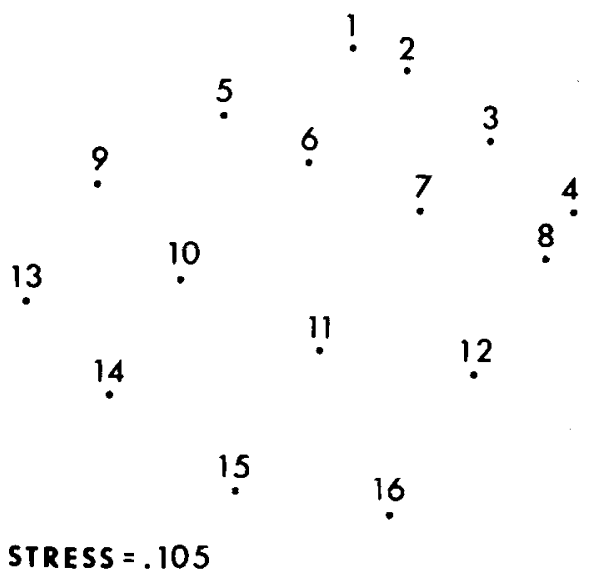

Figure 10. Best fitting scaling configuration for 16 width-height rectangles (Experiment 2). (This configuration is based on a random starting configuration, Euclidean metric, secondary approach to ties, and Stress Formula 1.)

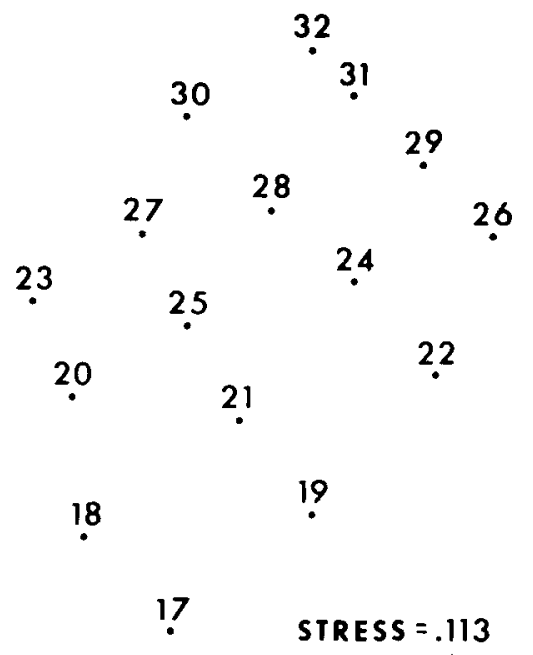

Figure 11. Best fitting scaling configuration for 16 area shape rectangles (Experiment 2). (This configuration is based on a random starting configuration, Euclidean metric, secondary approach to ties, and Stress Formula 1.) 
stimulus pairs corresponding to a given product structure, 10 scaling solutions were obtained for the Euclidean and city block metrics. In all cases, the Euclidean solution yielded lower stress values than the city block. For the width-height stimuli, the stress (Formula 1) values ranged from .193 to .356, with a median value of .336 for the city block metric, and from .105 to .128 , with a median value of .105 for the Euclidean metric. For the area shape stimuli, the stress values ranged from .146 to .363, median value .285 , for the city block metric; for the Euclidean metric, stress values rariged from .113 to .312 , median value .128 . Furthermore, since the theoretically most interpretable configurations were Euclidean scaling solutions, one can conclude that this metric provides a better fit to subjects' data than the city block one.

Multiple regression analysis. The superiority of the Euclidean metric over the city block metric may be due to the fact that the Euclidean metric is more robust under violations in metric assumptions. As in the first experiment, a second procedure was used to fit the two combination rules under consideration. The mean dissimilarity scores corresponding to the 120 stimulus pairs were regressed on the unidimensional dissimilarity scores. The multiple regression analysis was performed separately for each product structure and for each value of $r$.

Fitting the equations

$$
Y_{p}=w_{D 1} D_{1 i}+w_{D 2} D_{2 i^{\prime}}+c
$$

and

$$
\mathrm{Y}_{\mathrm{p}}{ }^{2}=\mathrm{w}_{\mathrm{D} 1} \mathrm{D}_{1 \mathrm{i}}{ }^{2}+\mathrm{w}_{\mathrm{D} 2} \mathrm{D}_{2 \mathrm{j}^{2}}{ }^{2}+\mathrm{c}
$$

by the method of least squares, we obtain

$$
Y_{p}=.61 W_{i}+.67 \mathrm{H}_{i^{\prime}}+1.38
$$

and

$$
\mathrm{Y}_{\mathrm{p}}{ }^{2}=1.11 \mathrm{~W}_{\mathrm{i}}{ }^{2}+1.10 \mathrm{H}_{\mathrm{i}^{\prime 2}}{ }^{2}+1.12
$$

for the width height structure, and

$$
Y_{p}=.37 S_{i}+.22 A_{i^{\prime}}+2.76
$$

and

$$
\mathrm{Y}_{\mathrm{p}}{ }^{2}=.64 \mathrm{~S}_{\mathrm{i}}{ }^{2}+.44 \mathrm{~A}_{\mathrm{i}^{\prime 2}}+6.77
$$

for the area shape product structure. For the widthheight structure, the multiple correlation coefficients are .923 and .922 for city block and Euclidean metrics (implying that $85 \%$ of the variance in both $\mathrm{Y}$ and $\mathrm{Y}^{2}$ values was explained). For the area shape product structure, the multiple correlation coefficients are .61 and .75 , implying that $37 \%$ of the variance in the $\mathrm{Y}$ values and $56 \%$ of the variance in the $\mathrm{Y}^{2}$ values was explained by the particular combination rule. These results indicate that the city block and Euclidean metrics provide equivalent approximations to a subject's rule of combination for the width-height stimuli, but that the Euclidean metric provides a better approximation for the area shape stimuli.

\section{Discussion}

Experiment 1 investigated two characteristics of subject's representations of highly analyzable stimuli: their multidimensional organization and their metric distances. In contrast, Experiment 2 examined that relationship in wholistic forms. The findings can be briefly summarized as follows: (a) There was an interaction between perceptual dimensions for both product structures. (b) The Euclidean metric provided a better fit to the proximity data than did the city block metric.

Two major analyses were employed to illustrate the perceptual interaction among dimensions. In the first analysis, an analysis of variance on interstimulus distances, the finding of a significant Stimulus Pairs effect indicated a violation of the prediction that stimulus pairs designated by the same interval notation should yield equal dissimilarity ratings. The significant interaction effect indicated that the ordering between intervals along one dimension was not invariant across intervals of the second dimension. In the multidimensional scaling analysis, the best fitting solutions to the proximity data were configurations of interacting dimensions-spatial arrangements of nonparallel lines consisting of points that varied on one attribute. Hence, it was concluded that width and height, as well as area and shape, combined in a nonadditive fashion to determine overall dissimilarity.

As for the brightness size stimuli, support for the Euclidean metric was more equivocal. The Euclidean metric yielded lower stress values and more interpretable configurations for both product structures than scaling solutions obtained under the city block metric. Since a similar result has previously been reported for the brightness size stimuli, where just the opposite result was predicted, this finding could be artifactual, and therefore a second procedure was used to fit a metric. For each product structure, bidimensional ratings were regressed on unidimensional ratings according to either an Euclidean or city block combination rule. The multiple correlation coefficients were identical in the width-height product structure, and the Euclidean metric yielded a higher coefficient in the area shape structure. Thus, although there is some evidence that the Euclidean metric is the true distance metric, it appears that 
metric structure is less discriminating than dimensional structure in differentiating analyzable from integral stimuli.

\section{Augmentation Effect and Dimensional Structure}

The findings provide a partial replication of the results reported by Krantz and Tversky (1975). Although they also report an interaction among perceptual dimensions, they were able to characterize this interaction as an augmentation effect for both product structures. The effect, however, was greatest for area shape. The most pronounced effect was the increased dissimilarity for a given pair of shapes as area increased. In contrast, in the present experiment, the interaction effect was interpretable for only width height, in which the width interval appeared larger, the more extreme the level of the height dimension.

If a systematic interaction effect is interpreted as a basic dimensional structure that must be modified to include an interaction component, then it appears that the two experiments differed in terms of the best fitting dimensional structure. Krantz and Tversky report that a majority of subjects (14 of 17) violated the width-height ordering prediction in favor of area shape in contrast to 8 of 17 subjects who violated the area shape ordering prediction in favor of width height. Since the width-height violations also tended to be larger than the area shape violations, they concluded that area shape provided a better account of the data even though neither dimensional structure was acceptable. The present experiment did not analyze systematic violations in one dimensional structure in terms of the alternative structure. However, the finding that an additive combination rule accounted for a greater proportion of variance in width-height ratings than for area shape, suggests that width height provided a better fit.

Our failure to isolate the same dimensional structure (i.e., area shape) as Krantz and Tversky is puzzling. At present, the discrepancy cannot be satisfactorily explained, but the following should be noted. (a) Krantz and Tversky analyzed only 62 out of the 92 possible stimulus pairs in their Width Interval by Height Interval design. Thus, it may have been more difficult to diagnose systematic deviations from the equality or ordering predictions in the width-height structure. (b) Krantz and Tversky reported a significant area illusion: Tall rectangles looked bigger than shorter ones of equal area. They therefore chose their rectangles to be squat (the largest height value was smaller than the smallest width value), and indicated that no variation in perceived area was subsequently observed within this range of squat shapes. In contrast, the present experiment provided no independent check of whether the effect had been successfully controlled.
If perceived area did vary as a function of height, then this might have introduced additional noise into the area shape design over and beyond that produced by interacting dimensions.

To summarize, multidimensional models do not satisfactorily explain the integration of rectangle attributes. The size illusions are attributed to some global stimulus property that presumably is no simple function of single stimulus dimensions (Cantor \& Thomas, 1977).

\section{GENERAL DISCUSSION}

\section{Integration of Perceptual Dimensions}

The present experiments analyzed several predictions made by multidimensional models in an attempt to infer how subjects perceive multivalued variation. The results indicated an additive combination rule of the city block type for brightness size stimuli, but a violation of interdimensional additivity for wholistic forms. It was concluded that brightness size stimuli are dimensionally organized while rectangular forms are not. But how, then, do subjects integrate physical dimensions for the case of unanalyzable stimuli?

It seems to the author that there are two solutions to this problem. If there is an interaction between attributes, then one can go on and investigate more complex combination rules of the type suggested by Krantz and Tversky, 1975 (e.g., Equation 6 in their paper.) The new combination rules take into account an interaction effect and, at the same time, maintain the attributes' status as psychological dimensions. Since interactions between perceptual dimensions ia a widespread phenomenon, it would seem that this approach has some merit. On the other hand, rejection of an additive combination rule excludes a large class of multidimensional scaling models, including the Euclidean and other Minkowski $r$ metrics.

A second approach would be to continue to maintain interdimensional additivity and intradimensional subtractivity as basic dimensional properties, and to search for more derived dimensions that satisfy them (Carroll \& Wish, 1974; Schönemann, 1977). In this approach, the subjective dimension is a more complicated mapping of the physical dimension, but the overall combination rule is still of the additive type. This second approach is adopted by most multidimensional scaling models which assume both additivity and subtractivity in the underlying dimensions. Thus, in the second experiment, the stimuli could be embedded in a two-dimensional Euclidean space, even though the physical dimensions were interacting. In the scaling configuration, this interaction between original attributes is reflected by nonparallel lines (as in Figures 10 and 11) and the 
introduction of additional dimensions.

If it can be assumed that the physical dimensions correspond to some underlying psychological process, then it would seem that the first approach is the preferred one. If, however, our major goal is the reduction of complex data, then the second procedure should be followed. In this case, however, the resulting dimensional structure may not necessarily correspond to the underlying psychological one.

\section{Dimensional vs. Metric Structures as Indicators of Analyzability}

Several considerations suggest that the concept of dimensional structure should take precedence over metric representation. First, since the Minkowski $r$ metrics are special cases of the additive difference model, dimensional organization is a logically more prior consideration than metric structure. Second, because selective attention occurs with highly analyzable stimuli, subjects might not succeed in attending to all multidimensional components at once (Shepard, 1964). Intra- and intersubject fluctuations in attention states might make metric fitting especially difficult for such stimuli, as well as lead to inaccurate estimates. Third, the best fitting metric becomes difficult to interpret if it is based on derived dimensions. This is because the dimensional structure obtained from multidimensional scaling may not necessarily be a valid approximation to the underlying psychological one. Fourth, metric fitting gives us only an overall picture of the metric's goodness of fit. In contrast, a dimensional analysis enables us to investigate interactive patterns in different regions of the stimulus space. Finally, the goal of finding the appropriate metric by obtaining scaling solutions separately for different $r$ is both expensive and time consuming. Since the problem of slow convergence and local minima are more likely for nonEuclidean metrics (Shepard, 1974), many more scaling runs are needed. (Arabie reports that for twodimensional solutions, one should use as many as 100 different starting configurations for each value of $r$ to insure a nonlocal minimum.) Thus, although nonmetric multidimensional scaling makes possible the fitting of a variety of non-Euclidean metrics, this approach may not be practical.

In summary, our results support a distinction between integral and analyzable stimuli in terms of dimensional organization. Extension of this technique to a variety of different stimuli and to more behavioral measures of proximity remains the next logical step.

\section{REFERENCE NOTE}

1. Lingoes. J. C., \& Roskam. E. E. A mathematical and empirical study of two multidimensional scaling algorithms. Michigan Mathematic Psychology Program Report No. MMPP 71-1, April 1971. University of Michigan, Ann Arbor.

\section{REFERENCES}

Arabie, P. A. Concerning Monte Carlo evaluations of nonmetric multidimensional scaling algorithms. Psychometrika, 1973, 38, $607-608$.

Attreave, F. Dimensions of similarity. American Journal of Psychology, 1950, 63, 516-556.

Beals, R., Krantz, D. H., \& Tversky, A. Foundations of multimensional scaling. Psychological Review, 1968, 75, $127-142$.

Bradley, J. V. Probability; decision; statistics. Englewood Cliffs, N.J: Prentice-Hall, 1976.

Cantor, N. E., \& Thomas, E. A. C. Control of attention in the processing of temporal and spatial information in complex visual patterns. Journal of Experimental Psychology: Human Perception and Performance, 1977, 3, 243-250.

CARRol. , J. D., \& Wish, M. Multidimensional perceptual models and measurement methods. In C. E. Carterette \& M.P. Friedman (Eds.), Handbook of perception: Psychophysical judgment and measurement (Vol. 2). New York: Academic Press, 1974.

Egeth, H. \& Pachella, R. Multidimensional stimulus identification. Perception \& Psychophysics, 1969, 5, 341-346.

Eriksen, C. W., \& HaKe, H. W. Multidimensional stimulus differences and accuracy of discrimination. Journal of Experimental Psychology, 1955, 50, 153-160.

Felfoldy, G. L. Repetition effects in choice reaction time to multidimensional stimuli. Perception \& Psychophysics, 1974, 15, 453-459.

GARNER, W. R., \& FELFOLDY, G. L. Integrality of stimulus dimensions in various types of information processing. Cognitive Psychology, 1970, 1, 225-241.

GotTwald, R. L., \& Garner, W. R. Effects of focusing strategy on speeded classification with grouping, filtering, and condensation tasks. Perception \& Psychophysics, 1972, 11, 179.182.

GOTTWALD, R. L., \& GARNER, W. R. Filtering and condensation tasks with integral and separable dimensions. Perception \& Psychophysics, 1975, 18, 26-28.

HANDEL, S. Classification and similarity of multidimensional stimuli. Perceptual and Motor Skills, 1967, 24, 1191-1203.

Handel, S., \& ImaI, S. The free classification of analyzable and unanalyzable stimuli. Perception \& Psychophysics, 1972, 12 , 108-116.

Hyman, R., \& Well, A. Judgments of similarity and spatial models. Perception \& Psychophysics, 1967, 2, 233-248.

ImaI, S. Classification of sets of stimuli with different stimulus characteristics and numerical properties. Perception \& Psychophysics, 1966, 1, 48-54.

IMAI, S., \& GARNER, W. R. Discriminability and preference for attributes in free and constrained classification. Journal of Experimental Psychology, 1965, 69, 596-608.

InDOW, T.. \& KanazaWA, K. Multidimensional mappting of Munsell colors varying in hue, chroma, and value. Joumal of Experimental Psychology, 1960, 59, 330-336.

InDow, T., \& Uchizono, T. Multidimensional mapping of Munsell colors varying in hue and chroma. Journal of Experimental Psychology, 1960, 59, 321-329.

KLAHR, D. A. A Monte Carlo investigation of the statistical significance of Kruskal's nonmetric scaling procedures. Psychometrika, 1969, 34, 319-330.

Krantz. D. H. Visual scaling. In D. Jameson \& L. M. Hurvich (Eds.), Visual psychophysics. Berlin: Springer, 1972.

Krantz, D. H., \& Tversky, A. Similarity of rectangles: An analysis of subjective dimensions. Journal of Mathematical Psychology, 1975, 12, 4-34.

KRUSKAL, J. B. Multidimensional scaling by optimizing goodness of fit to a nonmetric hypothesis. Psychometrika, 1964, 29, 1-27.

LINGOES, J. C. The Guttman-Lingoes nonmetric program series. Ann Arbor: Mathesis Press, 1973.

Lockhead, G. R., \& King, M. C. Classifying integral stimuli. Journal of Experimental Psychology: Human Perception and Performance, 1977, 3, 436-443. 
Monahan, J. S., \& Lockhead, G. R. Identification of integral stimuli. Journal of Experimental Psychology: General, 1977, 106, 94-110.

Newhall, S. M. Preliminary report on the O.S.A. Subcommittee on the spacing of the Munsell colors. Journal of the Optical Society of America, 1940, 30, 617-654.

Pomerantz, J. R., \& Garner, W. R. Stimulus configutation in selective attention tasks. Perception \& Psychophysics, 1973, 14, 565-569.

Pomerantz, J. R., \& Schwaitzberg, S. D. Grouping by proximity: Selective attention measures. Perception \& Psychophysics, 1975, 18, 355-361.

Schöneman, P. H. Similarity of rectangles. Journal of Mathematical Psychology, 1977, 16, 161-165.

SHEPARD, R. N. Attention and the metric structure of the stimulus space. Journal of Mathematical Psychology, 1964, 1, 54-87.

SHEPARD, R. N. Representation of structure in similarity data: Problems and prospects. Psychometrika, 1974, 39, 373-421.
Spence, I. A. A Monte Carlo evaluation of three nonmetric scaling algorithms. Psychometrika, 1972, 37, 461-486.

TVERSKY, A., \& Krantz, D. H. Similarity of schematic faces: A test of interdimensional additivity. Perception \& Psychophysics, 1969, 5, 124-128.

TVErsky, A., \& Krantz, D. H. The dimensional representation and the metric structure of similarity data. Journal of Mathematical Psychology, 1970, 7, 572-596.

WENDER, K. A test of the independence of dimensions in multidimensional scaling. Perception \& Psychophysics, 1971, 10 , 30-32.

WINER, B. J. Statistical principles in experimental design (2nd ed.). New York: McGraw-Hill, 1971.

(Received for publication March 14, 1978; revision accepted August 15, 1978.) 